\title{
Conditions for Propagation of Air Inflammation
}

\author{
Fedotov VG* and Fedotova EY \\ NN Semenov Institute of Chemical Physics of Russian Academia of Sciences, Russia
}

Received: 制: October 12, 2018; Published: 制: November 21, 2018

*Corresponding author: Fedotov VG, NN Semenov Institute of Chemical Physics of Russian Academia of Sciences, Moscow, Russian Federation

\begin{abstract}
Electronic Energy Explosion (EEE) in Air initiated by electric discharge near ferrite surface in the closed volume at the pressure exceeding 1.3 atm produces radiating active zone filling the entire reactor volume. This phenomenon is explained by propagation of Air inflammation at conditions mentioned above. It makes possible to inflame Air in any big closed volume.
\end{abstract}

Keywords: Electronic Energy; Explosion; Air; Absolute Pressure; NO; O(1s); Branched Chain Reaction; Inflammation; Propagation of Inflammation; Chemical Luminescence; Nitrogen Oxidation

Abbreviations: EEE: Electronic Energy Explosion; M: Molecules

\section{Introduction}

In the active medium of Electronic Energy Explosion, initiated by electric discharge near ferrite surface, takes place the nitrogen oxidation branched chain reaction [1,2]. The inverted populations of electronic states of several molecules formed in the chain reaction were observed at these conditions [3]. Moreover Laser generation and Dicke super-radiance were observed in the EEE active medium $[4,5]$. The air at atmospheric pressure was used in all the published experiments with EEE. The volume of EEE active medium at such conditions did not exceed 20-50 cubic centimeters. In the work [6] the transfer of inflammation from the EEE active medium to adjacent zones was observed at atmospheric pressure. Electric field with tension about $1000 \mathrm{~V} / \mathrm{cm}$ was needed for transfer the inflammation. However one should expect nitrogen oxides production (and laser generation energy) to be increased by increasing gas pressure and / or volume of the reaction zone. The Aim of this study was an attempt to perform the EEE at the pressure exceeding $1 \mathrm{~atm}$.

\section{Methods of Experiment}

A bottle of $1 \mathrm{~L}$ volume made of transparent plastic served as reactor in this work. The bottle was equipped with hermetically fitted cover. Through the holes in the cover we brought in the bottle two wires contacting with a piece of ferromagnetic dielectric of the type $3000 \mathrm{HMC}$ (nickel-manganese ferrite with $\mu=3000$, which is used for magnetic cores of TV fly back transformers). The piece of ferrite was hung on the wires inside the bottle. Another hole in the cover was drilled for the connecting pipe joined to the compressor. The fourth hole in the cover was used for connecting of the manometer. This arrangement enabled to achieve about 1.5 atm absolute air pressure in the bottle.The electrical circuit used for discharge initiation was described earlier [7]. It contains ACrectifier with output voltage $440 \mathrm{~V}, 2$ capacitors and a switch for connecting charged capacitors to the discharge gap [7]. A digital camera Sony DSC-650 was used for video recording of EEE in the bottle (Figure 1).

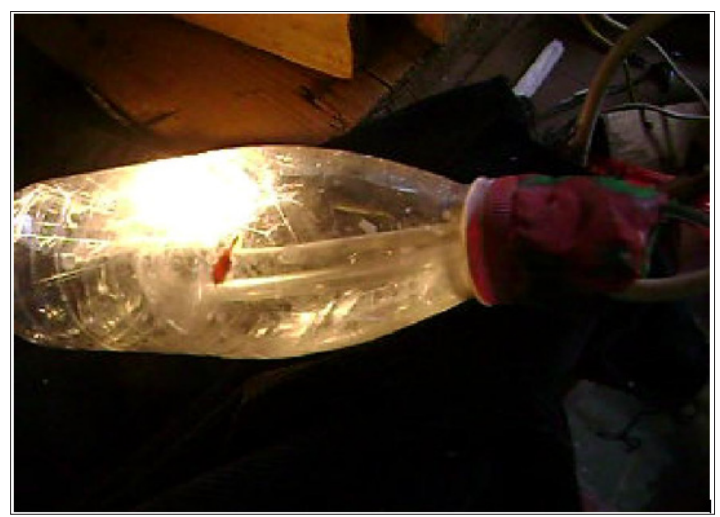

Figure 1: The video frame containing an image of EEE inside the bottle with air at atmospheric pressure.

\section{Results of Experiment}

A frame of video containing EEE image inside the bottle at atmospheric pressure is placed at Figure 1. It can be seen that the 
radiating active zone of EEE has the volume no more then $100 \mathrm{~cm}^{3}$ and is located close to the discharge gap. (The distance between the contact points of wires on the ferrite surface was approximately $4 \mathrm{~mm}$ ). The frame of video at Figure 2 contains the image of EEE inside the bottle at the pressure $1.3 \mathrm{~atm}$. In that case the radiating active zone filled the entire volume of the bottle (Figure 2).

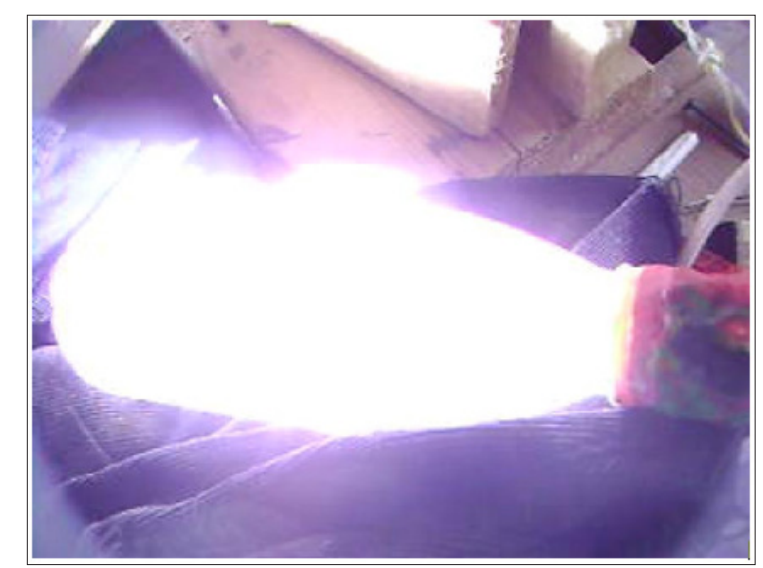

Figure 2: The video frame containing an image of EEE inside the bottle with air at pressure $1.3 \mathrm{~atm}$.

\section{Discussion}

In the previous experiments [1-4] the extinction of radiating active zone beyond the reach of electric field was observed. At the pressures higher then $1.3 \mathrm{~atm}$ the radiating zone filled up the entire volume of reactor; no extinction of radiation intensity with distance from the discharge gap was observed. An experiment with the reactor volume $10 \mathrm{~L}$ was performed and it showed the same result (at the air pressure more then $1.3 \mathrm{~atm}$ the EEE radiating zone filled the entire reactor volume $10 \mathrm{l}$ ). According to the Chemical Kinetic model of the nitrogen oxidation chain reaction [8] the chains branching rate is proportional to the factor $\mathrm{k}[\mathrm{NO}][\mathrm{M}]$, where $\mathrm{M}$ is the total concentration of molecules in the gas. It means that the chains branching rate rises proportional to the air pressure in the reactor. In the same way, NO concentration achieved in the reaction zone rises proportional M too. Transfer of $\mathrm{NO}$ and $\mathrm{O}(1 \mathrm{~S})$ atoms in the fresh air mixture results in development of chemical luminescent flash in it. This mechanism of inflammation propagation does not depend on the reactor volume. For this reason one can expect that the radiating active zone will fill the reactor of any big volume. So the amount of reaction products (nitrogen oxides) together with the production energetically effectiveness will rise unrestrictedly with enhancing of reactor volume.

\section{References}

1. Fedotov VG, Fedotova E Ya (2015) Explosion in atmospheric air initiated by electric discharge and associated with the growth of the concentrations of elwctronically excited molecules and free charges. Russian Journal of Physical Chemistry B 9(2): 223-227.

2. Fedotov VG, Fedotova E Ya (2015) The chain reaction of atmospheric nitrogen oxidation, initiated by electric discharge in air. J Physical Chem \& Biophys 5(6): 195.

3. Fedotov VG, Fedotova E Ya (2017) The inversed populations in the active medium of Electronic Energy Explosion (EEE) in the air. J Phys \& astron 5(1): 106.

4. Fedotov VG, Fedotova E Ya (2017) Multicolor laser generation Resulting from a Chain Reaction of Atmospheric Nitrogen Oxidation Initiated by an Electric Discharge in Air. Russian J of Physical Chem B 11(6): 928931.

5. Fedotov VG, Fedotova E Ya (2018) Superradiation (according to R. H. Dicke) in the Nitrogen Oxidation Chain Reaction. Open Access J of Science 2(3): 174-175.

6. Fedotov VG, Fedotova E Ya (2018) Transfer of inflammation in the Air. Open Access J of Science 2(1): 1-2.

7. Fedotov VG, Fedotova E Ya (2018) The Negative temperature coefficient for the Nitrogen Oxidation Chain Reaction and its Effect on Superluminescence Initiated by Electrical Discharge. Journal of Applied Physics \& Nanotechnol 1(1): 004.

8. Fedotov VG, Fedotova E Ya (2016) Chemical kinetic model of the chain reaction of atmospheric nitrogen oxidation initiated by electric discharge. Russian Journal of Physical Chemistry B 10(5): 748-752.
ISSN: 2574-1241

DOI: $10.26717 / B J S T R .2018 .11 .002074$

Fedotov VG. Biomed J Sci \& Tech Res

This work is licensed under Creative

Commons Attribution 4.0 License

Submission Link: https://biomedres.us/submit-manuscript.php

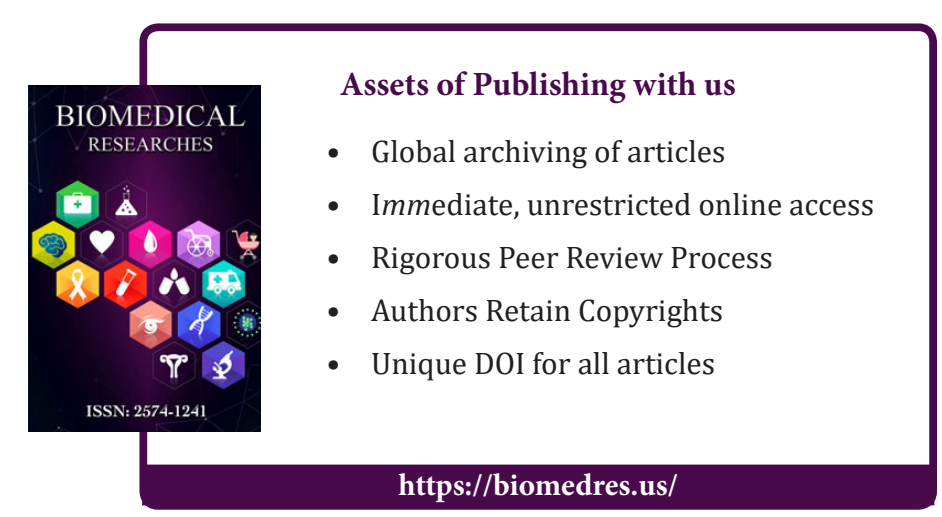

